\title{
A p.(Glu809Lys) Mutation in the WFS1 Gene Associated with Wolfram-like Syndrome: A Case Report
}

\author{
Dagmar Prochazkova1, Zuzana Hruba2, Petra Konecna1, Jarmila Skotakova3, Lenka Fajkusova2 \\ ${ }^{1}$ Medical Faculty of Masaryk University and University Hospital Brno, Department of Pediatrics, Brno, Czech Republic \\ 2 Medical Faculty of Masaryk University and University Hospital Brno, Department of Internal Medicine, Division of Hematology and Oncology, \\ Centre of Molecular Biology and Gene Therapy, Brno, Czech Republic \\ ${ }^{3}$ Medical Faculty of Masaryk University and University Hospital Brno, Department of Pediatric Radiology, Brno, Czech Republic
}

\section{ABSTRACT}

Wolfram-like syndrome (WFSL) is a rare autosomal dominant disease characterised by congenital progressive hearing loss, diabetes mellitus, and optic atrophy. The patient was a boy with the juvenile form of diabetes mellitus and findings which clinically matched the symptoms of Wolfram syndrome. At the age of $31 / 4$ years, diabetes mellitus was diagnosed in this boy who also had severe psychomotor retardation, failure to thrive, a dysmorphic face with Peters anomaly type 3 (i.e. posterior central defect with stromal opacity of the cornea, adhering stripes of the iris, and cataract with corneolenticular adhesion), congenital glaucoma, megalocornea, severe hearing impairment, a one-sided deformity of the auricle with atresia of the bony and soft external auditory canal, non-differentiable eardrum, missing os incus, hypothyreosis, and nephrocalcinosis. Molecular-genetic examinations revealed a de novo mutation p.(Glu809Lys) in the WFS1 gene. No mutations were detected in the biological parents. The mutation p.(Glu809Lys) in the WFS1 gene is associated with WFSL.

Keywords: Wolfram syndrome, genotype, phenotype

Conflict of interest: None declared

Received: 19.02 .2016

Accepted: 22.05 .2016

\section{Introduction}

Wolfram syndrome (WFS, OMIM 222300) is a rare genetic disease with a prevalence of cca. 1:710.000 (1). The disease is also known as the DIDMOAD syndrome (diabetes insipidus, diabetes mellitus, optic atrophy, deafness). Most of the individuals afflicted with this disease have the recessive mutation in the WFS gene 1 (WFS1, 4p16.3) $(1,2)$ and rarely in the WFS gene 2 (WFS2) (3). A dominant mutation in the WFS1 gene was also described in connection with sensorineural hearing loss, deafness, and optic atrophy [Wolfram-like syndrome (WFSL)] (4).
The novelty of the data and their impact on the field: the p.(Glu809Lys) mutation in the WFSI gene is associated with the occurrence of the WFSL.

\section{Address for Correspondence}

Dagmar Prochazkova MD, Medical Faculty of Masaryk University and University Hospital Brno, Department of Pediatrics, Brno, Czech Republic Phone: +420 532234962 E-mail: prochazkovad@fnbrno.cz

OJournal of Clinical Research in Pediatric Endocrinology, Published by Galenos Publishing. 


\section{Case Report}

Our patient was a boy whose clinical findings matched those reported for the Wolfram syndrome. At the age of 3 1/4 years, the patient was diagnosed to have diabetes mellitus along with severe psychomotor retardation, failure to thrive, a dysmorphic face with Peters anomaly (PS) type 3 (posterior central defect with stromal opacity of the cornea, adhering stripes of the iris, and cataract with corneolenticular adhesion), congenital glaucoma, megalocornea, hypothyreosis, nephrocalcinosis, severe hearing impairment, a one-sided deformity of the auricle with atresia of the bony and soft external auditory canal, a non-differentiable eardrum, and a missing os incus. At presentation, the patient did not have optic atrophy or diabetes insipidus. The proband's karyotype was 46,XY, 9qh+.

We performed sequencing analysis of the coding exons and adjacent intron regions of the WFS1 gene and of the B3GALTL, CYP1B1, PITX2, and PAX6 genes associated with PS. The analysis for the WFS1 gene was complemented by multiple ligation-dependent probe amplification (SALSA MLPA P163 GJB-WFS1, MRC-Holland) identifying potential deletions/ duplications. Apart from standard polymorphisms, the only potentially causal mutation was found to be a heterozygous missense mutation in the WFS1 gene, namely, c.2425G>A, p.(Glu809Lys). This mutation was analysed in the proband's family, namely, in both parents and siblings of the proband-twin $B$. The mutation was not detected in any of the family members and the final diagnosis was WFSL caused by a de novo mutation c.2425G >A, p.(Glu809Lys). This mutation localised in the exon 8 of WFS1 was not reported in databases (http://databases. lovd.nl/whole_genome/genes or http://exac.broadinstitute.org/) and was described as a likely pathogenic finding in http://www. ncbi.nlm.nih.gov/clinvar/variation/215413/. In silico analysis of this mutation using prediction programs PolyPhen-2 and SIFT showed probable damaging and damaging effects, respectively.

\section{Discussion}

In 2014, Matsunaga et al (1) described a proband with WFS and c.2425G >A, p.(Glu809Lys) mutation in the WFS1 gene. The patient suffered from diabetes mellitus, optic atrophy, deafness, and mental disorder. In the same year, Lee et al (5) mentioned a proband with c.2425G>A, p.(Glu809Lys) mutation in the WFS1 gene. The proband suffered from cataract, hypotonia, sensorineural deafness, and diabetes mellitus. The second causal mutation was not determined in either patient. Follow- up molecular genetic examinations of the family members had apparently not been carried out in these cases. We believe that molecular genetic examination in the members of the family of a proband with one mutation in the WFS1 gene is important.

\section{Acknowledgments}

Our thanks go to the Department of Pediatric Otorhinolaryngology University Hospital Brno and the Department of Pediatric Ophthalmology University Hospital Brno for the care of our patient.

\section{Ethics}

Informed Consent: It was taken.

Peer-review: Externally peer-reviewed.

\section{Authorship Contributions}

Concept: Dagmar Prochazkova, Design: Dagmar Prochazkova, Lenka Fajkusova, Data Collection or Processing: Zuzana Hruba, Petra Konecna, Jarmila Skotakova, Analysis or Interpretation: Dagmar Prochazkova, Lenka Fajkusova, Literature Search: Dagmar Prochazkova, Lenka Fajkusova, Writing: Dagmar Prochazkova.

Financial Disclosure: This work was funded by the project of the Internal Grant Agency of the Czech Ministry of Health (NT/14574-3) and the project of the Czech Ministry of Health for conceptual development of research organization 65269705 (University Hospital Brno, Brno, Czech Republic).

\section{References}

1. Matsunaga $\mathrm{K}$, Tanabe $\mathrm{K}$, Inoue $\mathrm{H}$, Okuya $\mathrm{S}$, Ohta $\mathrm{Y}$, Akiyama $\mathrm{M}$, Taguchi A, Kora Y, Okayama N, Yamada Y, Wada Y, Amemiya S, Sugihara $S$, Nakao $Y$, Oka $Y$, Tanizawa $Y$. Wolfram syndrome in the Japanese population; molecular analysis of WFS1 gene and characterization of clinical features. PLoS One 2014;9:106906.

2. Nalbantoglu Elmas O, Demir K, Soylu N, Celik N, Ozkan B. Importance of insulin immunoassays in the diagnosis of factitious hypoglycemia. J Clin Res Pediatr Endocrinol 2014;6:258-261.

3. Inoue H, Tanizawa Y, Wasson J, Behn P, Kalidas K, Bernal-Mizrachi E, Mueckler M, Marshall H, Donis-Keller H, Crock P, Rogers D, Mikuni M, Kumashiro H, Higashi K, Sobue G, Oka Y, Permutt MA. A gene encoding a transmembrane protein is mutated in patients with diabetes mellitus and optic atrophy (Wolfram syndrome). Nat Genet 1998;20:143-148.

4. Valero R, Bannwarth $\mathrm{S}$, Roman $\mathrm{S}$, Paquis-Flucklinger V, Vialettes B. Autosomal dominant transmission of diabetes and congenital hearing impairment secondary to a missense mutation in the WFS1 gene. Diabet Med 2008;25:657-661.

5. Lee YM, Abdullah RAR, Keng WT. Wolfram syndrome and diabetes. J Clin Endocrinol Metab 2014;4(Suppl 1):21. 